\title{
Molecular Imaging in Cardiovascular Diseases
}

\section{Molekulare kardiovaskuläre MRT-Bildgebung}

Authors

Affiliations
R. M. Botnar ${ }^{1}$, H. Ebersberger ${ }^{2}$, D. Noerenberg ${ }^{3}$, C. H. P. Jansen ${ }^{1}$, A. J. Wiethoff ${ }^{1}$, A. Schuster ${ }^{1}$, M. Kasner ${ }^{4}$, T. C. Walter ${ }^{5}$, G. Knobloch ${ }^{3}$, P. Hoppe ${ }^{6}$, G. Diederichs ${ }^{3}$, B. Hamm ${ }^{3}$, M. R. Makowski ${ }^{3}$

Affiliation addresses are listed at the end of the article.
Key words

- MR-imaging

- arteriosclerosis

- gadolinium received $\quad 7.1 .2014$

accepted $\quad 8.9 .2014$

Bibliography

DOI http://dx.doi.org/

10.1055/s-0034-1385451

Published online: 13.1.2015

Fortschr Röntgenstr 2015; 187:

92-101 @ Georg Thieme Verlag

KG Stuttgart · New York .

ISSN 1438-9029

\section{Correspondence}

Dr. Marcus R. Makowski

Charité, Institut für Radiologie

Charitéplatz 1

10115 Berlin

Germany

Tel.: ++49/030/450627345

Fax: ++49/030/4507527911

marcus.makowski@charite.de

\section{Abstract \\ $\nabla$}

Cardiovascular diseases remain the leading cause of morbidity and mortality in industrialized and developing countries. In clinical practice, the in-vivo identification of atherosclerotic lesions, which can lead to complications such as heart attack or stroke, remains difficult. Imaging techniques provide the reference standard for the detection of clinically significant atherosclerotic changes in the coronary and carotid arteries. The assessment of the luminal narrowing is feasible, while the differentiation of stable and potentially unstable or vulnerable atherosclerotic plaques is currently not possible using non-invasive imaging. With high spatial resolution and high soft tissue contrast, magnetic resonance imaging (MRI) is a suitable method for the evaluation of the thin arterial wall. In clinical practice, native MRI of the vessel wall already allows the differentiation and characterization of components of atherosclerotic plaques in the carotid arteries and the aorta. Additional diagnostic information can be gained by the use of non-specific MRI contrast agents. With the development of targeted molecular probes, that highlight specific molecules or cells, pathological processes can be visualized at a molecular level with high spatial resolution. In this review article, the development of pathophysiological changes leading to the development of the arterial wall are introduced and discussed. Additionally, principles of contrast enhanced imaging with non-specific contrast agents and molecular probes will be discussed and latest developments in the field of molecular imaging of the vascular wall will be introduced.
Key Points:

- Molecular magnetic resonance imaging has great potential to improve the in vivo characterization of atherosclerotic plaques.

- Based on the molecular information is feasible to enable a better differentiation of stable and unstable (vulnerable) atherosclerotic plaques.

Citation Format:

- Botnar RM, Ebersberger H, Noerenberg D etal. Molecular imaging in cardiovascular diseases. Fortschr Röntgenstr 2015; 187: 92-101

\section{Zusammenfassung}

$\nabla$

Kardiovaskuläre Erkrankungen sind nach wie vor die häufigste Ursache für Morbidität und Mortalität in den westlichen Industrieländern, aber auch in Entwicklungsländern. Im klinischen Alltag bleibt die In-vivo-Identifizierung von arteriosklerotischen Läsionen, die zu Komplikationen wie Herzinfarkten oder Schlaganfällen führen können, weiterhin schwierig. Bildgebende Verfahren sind gegenwärtig der Referenzstandard für die Detektion klinisch relevanter arteriosklerotischer Veränderungen der Koronararterien und der Karotiden. Dabei bleibt die Beurteilung auf die bildmorphologische Lumeneinengung beschränkt, während eine Differenzierung von stabilen und potenziell instabilen oder vulnerablen arteriosklerotischen Plaques aktuell im Rahmen der nichtinvasiven Bildgebung nicht möglich ist. Mit hoher räumlicher Auflösung und hohem Weichteilkontrast ist die Magnetresonanztomografie (MRT) eine gut geeignete Methode zur Darstellung und Beurteilung der dünnen Arterienwand. In der klinischen Praxis erlaubt die native MRT der Gefäßwand bereits eine Differenzierung sowie Charakterisierung von Komponenten arteriosklerotischer Plaques der Karotiden und der 
Aorta. Ein Zugewinn an diagnostischer Information kann durch den Einsatz zugelassener unspezifischer MRT-Kontrastmittel erreicht werden. Mit der Entwicklung von zielgerichteten molekularen Sonden, die bestimmte Moleküle oder Zellen markieren, lassen sich pathologische Prozesse auf molekularer Ebene mit hoher räumlicher Auflösung darstellen. In diesem Übersichtsartikel werden zunächst die zur Entwicklung der Arteriosklerose führenden pathophysiologischen Veränderungen der arteriellen Gefäßwand erläutert. Es folgt eine Darstellung der Wirkweise sowie der Eigenschaften unspezifischer Kontrastmittel sowie molekularer Sonden für die MRT-Bildgebung. Zusätzlich werden die neuesten Entwicklungen auf dem Gebiet der molekularen Bildgebung der Gefäßwand erörtert.

\section{Introduction}

Cardiovascular diseases remain the leading cause of morbidity and mortality in industrialized and developing countries [1]. In particular, coronary artery diseases are the cause of the majority of events leading to cardiovascular mortality and morbidity[1]. In clinical practice, early detection of unstable (vulnerable) atherosclerotic lesions that can lead to these complications remains difficult. Various studies have demonstrated that a large proportion of ruptured plaques in the vascular regions with a less than $60 \%$ degree of stenosis can be found [2-4]. Therefore there is significant clinical interest in early detection of such plaques. New imaging procedures are required which will provide information regarding plaque composition and disclose biological processes which indicate the progression and destabilization of plaques. Angiographic imaging methods that can only assess lumen diameter remain the gold standard for the diagnosis of clinically significant stenosis of coronary and carotid arteries. Even severe atherosclerotic changes in the arterial wall can escape detection by conventional angiography, since in the context of atherosclerotic plaque development, such changes can result in positive remodeling, i.e. compensatory enlargement of the arterial cross section without significant lumen constriction [5]. Various studies have shown that atherosclerotic plaques exhibiting positive remodeling are associated with an increased risk of rupture [6]. In clinical application, native MRI can differentiate among the morphological components of atherosclerotic plaques in the carotids, the aorta as well as coronary arteries [7 - 9]. Additional information regarding plaque composition can be obtained by administering clinically-approved non-specific contrast agents [10]. Specific molecular probes that selectively highlight certain molecules or cells allow the visualization and characterization of pathological processes on the molecular level, thus enhancing the detection of early stages of disease, and improving treatment planning and therapy monitoring.

\section{Pathophysiology of atherosclerotic plaques}

Atherosclerotic changes in the arterial wall develop over a long period, and symptoms first appear when arterial blood flow is reduced due to plaque size, or when the plaque ma- trix is exposed after a rupture or as a consequence of surface erosion [11]. The gradual increase in plaque size does not represent a critical event, and in most cases can be adequately treated therapeutically before acute symptoms arise. On the other hand, plaque rupture represents a highly acute event that cannot be predicted with current imaging methods, and which can result in severe consequences such as thrombotic vessel occlusion and ischemia [12]. Pathological changes in the arterial wall in the context of atherosclerosis primarily affect the vascular endothelium. During the onset as well as development of atherosclerosis, there is an influx of modified lipids such as low-density lipoproteins (LDL), macrophages and smooth muscle cells [13]. Infiltration and depositing of LDL in the vascular endothelium is considered a precursor of atherosclerosis [13]. Oxidized LDL activates endothelial cells which in turn express adhesion molecules such as vascular cell adhesion molecule 1 (VCAM-1) and E-selectin. Activation of the endothelium leads to increased accumulation of monocytes and other inflammatory cells which play a significant role in all stages of atherosclerosis. If the vascular endothelium is affected by atherosclerosis, the result is a differentiation of monocytes and macrophages which consume modified LDL particles. In the course of this process, macrophages develop into cholesterol-accumulating foam cells. In this stage of plaque development, these lesions are called "fatty streaks". This type of lesion can be identified in the arterial wall of very young patients [13]. Further, activated macrophages release inflammatory cytokines, matrixmetalloproteinases as well as tissue factors and thus contribute to the progression and potential destabilization of atherosclerotic lesions [13]. Additionally in this stage, smooth muscle cells migrate from the media into the endothelium. These proliferating smooth muscle cells represent an essential component of the neointima and are responsible for the expression of extracellular matrix proteinases such as elastin and collagens [14]. In the course of continued plaque progression, hypoxia increasingly becomes more important, since greater blood flow is required due to elevated oxygen consumption. Hypoxic states contribute to the apoptosis of inflammatory cells and the proliferation and ingrowth of new blood vessels into the atherosclerotic vessel wall, a process also called neoangiogenesis [15]. It has been shown that, in comparison to stable lesions, unstable atherosclerotic plaques exhibit increased density on newly formed vessels (neoangiogenesis) [15]. When these fragile new blood vessels rupture, erythrocytes enter the matrix of atherosclerotic plaques due to blood penetration. If the cholesterol released from the erythrocyte membranes accumulates, the result is precipitation and crystallization which contribute to the accretion of one or more lipid-rich necrotic cores [16]. Eccentric growth in size of the atherosclerotic arterial wall can occur to compensate for the narrowing of the lumen caused by intimal thickening. This type of vessel wall alteration is called positive remodeling [5]. In the course of continuous abatement of inflammatory processes and depositing of modified lipids, the lipid cores can expand further, while at the same time proteinases degrade extracellular matrix proteins. This results in thinning of the fibrous cap and consequently to an increased risk of rupture [14]. When plaque ruptures, blood from the arterial lumen comes into contact with the plaque matrix and tissue factor, thus activating the coagulation cas- 
cade. This leads directly to accumulation and activation of platelets at the lesion site via binding to subendolethial collagens via constitutively expressed GPVI receptors, resulting in thrombus formation triggered by thrombin. If the newly formed thrombus causes narrowing or blockage of an artery, the consequence can be an acute myocardial infarction or stroke [13]. Various studies have shown that atherosclerotic lesions with a high risk of rupture (vulnerable plaques) exhibit the following primary characteristics: High plaque volume, a large necrotic core, thin fibrous cap as well as positive vascular wall remodeling $[17,18]$. It has been further indicated that vulnerable plaques are the trigger of the majority of acute symptomatic coronary artery events. In particular, plaque erosion on the endothelial surface even in early stages can lead to thrombus formation [19]. In the course of atherosclerotic plaque formation, deposition of hydroxyapatite results in calcification of the vascular wall. Intramural calcification in the atherosclerotic vascular wall is an active process exhibiting parallels to physiological bone formation. Various cellular and subcellular factors, including osteoblast-like cells, are instrumental in the actuation and progression of this process [20]. Typically calcification starts in the region of the lipid core within atherosclerotic plaque [21]. The apoptosis of migrated cells is directly related to the initiation and progression of plaque calcification [22]. Extracellular matrix proteins (e.g. collagens) are additionally involved in this process [23]. Despite some commonalities in the pathogenesis, a distinction must be made between the development of atherosclerosis in the coronary and carotid arteries. The differences between the two vascular territories are based primarily on the higher flow velocities and shear forces returned to the carotids. Thus plaque ulcerations and bleeding are more frequently found in the carotid arteries, whereas plaque erosion is more frequently found in the coronary arteries [24]. Calcification frequency does not differ significantly in both vascular territories, while calcified nodules (irregular nodules of calcium deposits) have been more frequently observed in cases of atherosclerotic changes in the carotid arteries [24]. A relationship between plaque rupture and symptomatic atherosclerosis has been demonstrated for both vascular territories; however, the degree of lumen narrowing does not always correlate with the presence of vulnerable plaque.

\section{Contrast-enhanced and molecular magnetic resonance imaging \\ $\nabla$}

\section{Principles of contrast-enhanced MRI}

Using molecular MRI employing targeted probes, biological processes can be non-invasively demonstrated on the molecular level. Unlike other imaging modalities such as single photon emission computed tomography (SPECT) or positron emission tomography (PET), MRI offers high physical resolution for identifying and assessing contrast agent distribution within the vascular wall $[11,25]$. Compared to SPECT or PET, a higher local concentration of MR contrast medium (e.g. Gd-DTPA) is required for reliable in vivo assessment. Various targeted MR contrast agents are used for molecular imaging, including probes based on gadolinium or iron oxide particles. The signal intensity of MRI is prima- rily determined by the local longitudinal (T1) and transverse (T2) relaxation time of free water protons. The functionality of the majority of MR contrast agents depends upon a shortening of these relaxation times. Gadolinium or iron oxide-based MR contrast media are therefore indirectly detected due to their effect on water protons. Specific receptors, molecules or cells can be visualized via active or passive mechanisms with the aid of molecular MRI contrast agents. Selective binding of the molecular probe to specific receptors or proteins (active targeting) represents one possibility for visualization. A further possibility for enhancement is based on accumulation of the probe due to certain characteristics of the pathological tissue (e.g. fibrosis) or specific cell types (e.g. macrophages resulting from phagocytosis). An example for passive targeting is provided by iron oxide particles consumed by macrophages [26].

\section{T1-shortening contrast agents and molecular probes}

Contrast agents and molecular probes causing shortening of the $\mathrm{T} 1$ relaxation time are primarily based on chelate complexes with trivalent gadolinium at the center. The existing seven unpaired electrons as well as the symmetry of the electronic states of chelate complexes with trivalent gadolinium result in a significant lowering of spin-lattice relaxation time [27]. The relaxivity of gadolinium-based contrast media depends upon various factors such as water exchange rate, hydration number, as well as the rotational correlation time [27]. The T1-shortening effect of gadolinium-based contrast agents and molecular probes is stronger than their T2-shortening effect [28]. Thus these bonds belong to "positive" MRI contrast agents; their effect is expressed as an increase of signal intensity on T1-weighted images. It has been shown that for T1-weighted gradient echo sequences, signal intensity across a wide concentration range is proportional to the local contrast agent concentration $[29,30]$. If, however, the local contrast agent concentration is very high, a noticeably reduced $\mathrm{T} 2$ relaxation time additionally occurs, $[29,30]$ together with a deviation from linear signal behavior. Depending upon the composition of the contrast medium or molecular probe, the relaxation effect additionally varies with the strength of the external magnetic field. An important factor in this depends upon whether the contrast medium is in the extracellular region, is bound to a receptor, or is absorbed intracellularly [31, 32]. T1-shortening MR contrast agents can, for example, be synthesized through conjugation of small ligands (e.g. small molecules, peptides, antibodies or glucose) with gadolinium chelate. The specific bond of such a probe to static or slowly-moving target structures leads to increased relaxivity, due to the so-called RIME effect (receptor-induced magnetization enhancement) [33]. An example of a contrast agent with this operating principle is gadofosveset trisodium. This medium binds reversibly to plasma albumin, resulting in a significant increase in relaxivity, thus allowing more sensitive in vivo detection [27]. In order to increase the sensitivity of the detection of molecular target structures that appear only in limited quantities, multimer-based contrast agents can be employed, for example. The T1 effect of the contrast medium can be enhanced by increasing the quantity of gadolinium chelates per molecular probe [34]. One example of such a probe is EP-2104 $\mathrm{R}$ (EPIX Pharmaceuticals, Lexington, USA). This fibrin-specific 
molecular probe has been successfully tested on patients for the disclosure of fibrin-rich thrombi. EP-2104 consists of a short peptide (11 amino acids) bound to four DOTA chelates [35]. An additional approach to increasing the relaxivity of contrast agents is the utilization of substances that assemble complexes on their own. Lipid-hydrofluorocarbon emulsions have been used as a platform to create these types of molecular probes. The specificity of these nanoparticles can be produced, for example, by the integration of a lipid-labeled biotin-avidin antibody complex in the particle. Hydrofluorocarbon nanoparticles have been loaded with up to approx. 90, 000 gadolinium atoms / $200 \mathrm{~nm}$ particles and successfully used for in vivo imaging of fibrin as well as $\alpha_{v \beta 3}$-integrin for atherosclerosis [36, 37]. Glucose, proteins and aptemers are additional ligands that can be used for the production of molecular MR contrast agents.

The following should be considered with respect to the translation of pre-clinically tested approaches into clinical practice. In contrast to doses of contrast agents in quantities permitted in humans, pre-clinical studies employ significantly higher dosages of the molecular probe. These high doses can affect cellular and molecular processes in the target structures. Further, in pre-clinical studies, generally high-field MRI scanners are used with corresponding test protocols. Again, in this instance, it can be difficult to translate these protocols directly into human applications.

\section{T2-shortening contrast agents and molecular probes}

MR contrast agents and molecular probes causing shortening of the $\mathrm{T} 2 / \mathrm{T} 2 *$ relaxation time are primarily based on iron oxide particles. When this particle type is used, the T2-shortening effect is greater than its T1 effect. The contract effect of iron oxide particles is thus negative, i.e. resulting in a signal loss in $\mathrm{T} 2 / \mathrm{T} 2{ }^{*}$-weighted images [38]. Compared to gadolinium-based contrast media and probes, iron oxide particles typically have a stronger effect on the relaxation time; therefore they can be detected with greater sensitivity [39]. As in the case of gadolinium-based contrast agents, the relaxation effect depends on the strength of the magnetic field used. Synthesis of stable iron oxide particles to achieve $\mathrm{T} 2$ / $\mathrm{T} 2 *$ contrast has been intensively investigated in recent years. On the whole, iron oxide particles can be subdivided into fractions with short as well as long blood half-life. Particles with short blood half-life include superparamagnetic iron oxide particles (SPIOs) such as ferumoxide. In solution, these particles tend to aggregate, and in the organism they are rapidly assumed by cells of the mononuclear phagocyte system. This type of particle is primarily used for liver imaging. Iron oxide particles with long blood half-life include, for example, ultra-small superparamagnetic iron oxide particles (USPIOs), such as ferumoxtran or ferumoxytol. Due to their stable coating, they remain monodisperse in solution, i.e. all particles are the same size. These particles are preferably employed for molecular imaging of patients with atherosclerosis, myocardial infarction or tumors. Depending on their size and composition, iron oxide particles have varying degrees of effect on R1 and $\mathrm{R} 2$ relaxation. SPIO particles have a stronger $\mathrm{R} 2$ effect than R1 effect. Therefore SPIO particles can be most sensitively visualized using T2 or T2*-weighted sequences [40]. For USPIO particles, the R1 and R2 effect is about the same. Thus they can likewise be visualized with T1-weighted se- quences using "positive" contrast [41]. In addition to the R1 and R2 effect, accumulation of SPIOs and USPIOs results directly in a disturbance of the magnetic effect $(\triangle B 0)$, resulting in the formation of a susceptibility gradient and frequency shift. The resulting spin dephasing can detected most sensitively with $\mathrm{T} 2{ }^{*}$-weighted sequences. Additional types of MR contrast agents and molecular probes whose contrast behavior depends on other mechanisms have been investigated and characterized; however, a discussion of them would be outside the focus of this article.

\section{Molecular vascular wall imaging \\ $\nabla$}

\section{Targeted molecular probes for displaying}

\section{plaque components}

In the course of plaque progression, smooth muscle cells and macrophages express extracellular matrix proteins, including elastin and collagen which represent an essential component of atherosclerotic plaques [14]. Recently an elastin-specific low-molecular probe was introduced and described. This probe is characterized by a rapid extravasation from the blood pool [42, 43]. Surface measurements based on the use of these molecular probes correlate with the plaque load in an apoE $\mathrm{E}^{-1}$ mouse with atherosclerotic vascular wall alterations ( $\bullet$ Fig. 1 ) [42]. In a porcine model with coronary remodeling after stent implantation used to simulate clinical imaging conditions, this molecular probe was successfully tested for in vivo assessment of the remodeling of the coronary vascular wall. The area of the contrast agent-enhanced measured in vivo displayed good correlation with the histologically determined extent of the remodeling [44]. A further example of a molecular probe with a bond to extracellular plaque components is gadofluorine. This is a gadolinium-based macrocyclic molecular probe with both hydrophilic and hydrophobic characteristics as well as a blood half-life comparable to conventional gadolinium chelates [45]. Heavy accumulation of gadofluorine in lipid-rich plaques was exhibited in a rabbit model with atherosclerosis [46]. Furthermore, recent publications have described co-localization of this molecular probe in regions with a high density of newly-formed vessels (a characteristic of vulnerable plaque) as well as collagen-rich (fibrous) plaque regions $[47,48]$. Fibrin is an additional protein that plays a role in the development and progression of atherosclerotic plaques [49]. It also has a key function in thrombus formation following plaque rupture. In various studies a fibrin-specific low-molecular probe has been successfully employed for imaging thrombi in the jugular vein, aorta, pulmonary arteries as well as in coronary arteries [ 35 , $36,50,51]$. Compared to conventional compounds such as Gd-DTPA, the probe indicated high sensitivity for the detection of fibrin; this is explained by the high relaxivity of the specific molecular probe. This specific molecular probe allowed selective demonstration of a coronary in-stent thrombus in an experimental animal subject ( $\bullet$ Fig. 2) [35]. The applied dose of $4-7.5 \mu \mathrm{mol} / \mathrm{kg}$ was significantly lower than the typical non-specific gadolinium-based MR contrast agent dose of $0.1-0.2 \mathrm{mmol} / \mathrm{kg}$. Finally, this molecular probe was successfully used for detecting thrombi in the aorta, carotid and coronary arteries in a patient; this dem- 


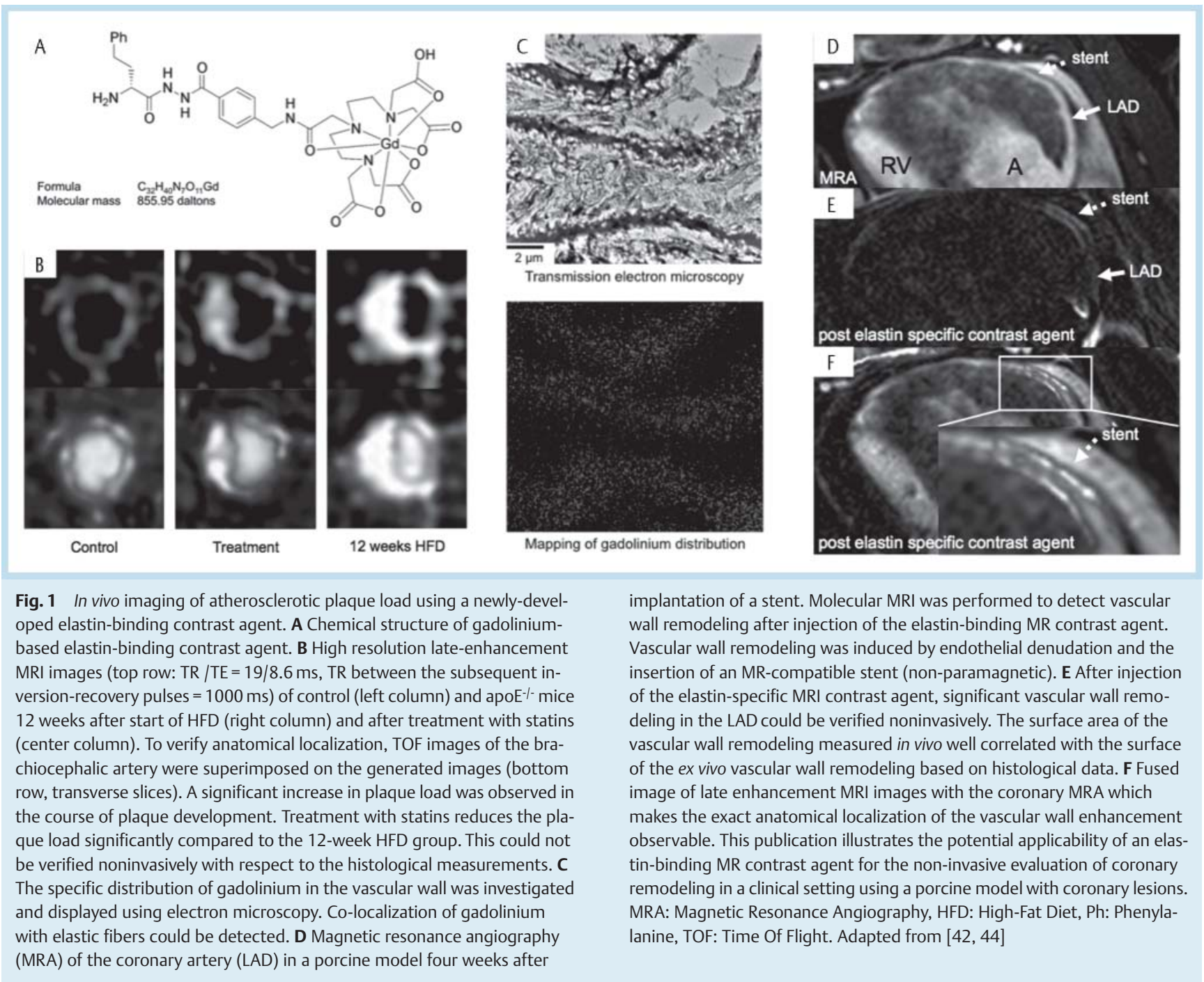

onstrated that clinical translation of targeted gadoliniumbased molecular MR contrast agents is possible [52]. Larger nanoparticles with more than 50, 000 gadolinium atoms per particle have likewise been successfully employed for in vivo imaging of a jugular thrombus in animals; however, this has not been translated to human application [36].

\section{Targeted molecular probes for displaying cellular surface} molecules

Endothelial cell adhesion molecules such as E-selectin, ICAM-1 (intercellular adhesion molecule-1) and VCAM-1 (vascular cell adhesion molecule-1) represent specific biomarkers of early atherosclerotic alterations. These molecules play an important role in endothelial activation and subsequent transmigration of inflammatory cells into the neointima [53]. The imaging of endothelial adhesion molecules has received much attention since these molecules are heavily expressed in the early stages of atherosclerotic plaque development. In addition, these molecules are located on the vessel endothelium and are readily accessible to intravenously applied molecular probes. In vivo detection of VCAM-1 has already been demonstrated on an animal subject with atherosclerosis. Significant VCAM-1-specific na- noparticle binding in the aortic root has been demonstrated using T2*-weighted MRI sequences( $\bullet$ Fig. 3) [54].

\section{Molecular probes for displaying macrophages}

In cases of atherosclerosis, the immune system responds with local inflammation to extracellular deposits of various lipid and cholesterol compounds [13]. Consequently, migration occurs among inflammatory cells such as monocytes which in the vascular wall differentiate into macrophages. High macrophage density in the fibrous cap is one of the accepted characteristics of vulnerable atherosclerotic plaques [13]. Different contrast agent approaches to displaying macrophages have been studied. The method most commonly investigated in animals and patients is based on the use of iron oxide particles with various coatings such as dextran or citrate [55]. The exact mechanism by which iron oxide particles enter atherosclerotic plaques has not yet been fully explained. A possible mechanism is based on the fact that iron oxide particles are initially phagocytized by intravascular monocytes, and that these iron-enriched monocytes then migrate into areas with high local inflammatory activity (e.g. into the plaque shoulder). Alternatively, iron oxide particles could enter atherosclerotic plaques due to increased endothelial permeability or via fragile newly- 

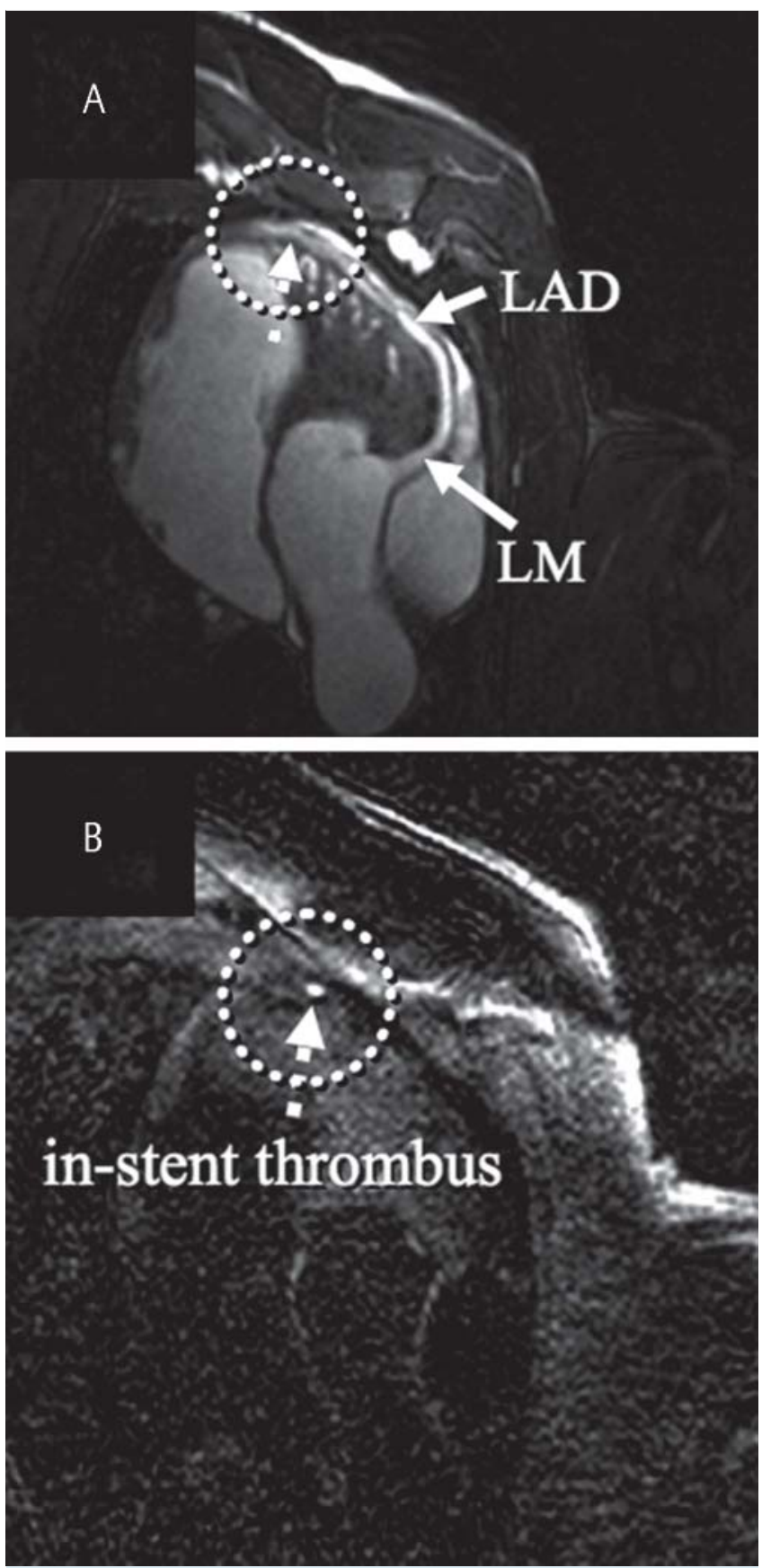

Fig. 2 In vivo illustration of an in-stent thrombus after injection of a fibrinbinding contrast agent. A gadolinium-based fibrin-binding contrast agent (EP-2104R) was injected into a porcine model with an in-stent thrombosis. D Magnetic resonance angiography (MRA) of the coronary artery (LAD) in a porcine model. Since a non-paramagnetic stent was used, no stent artifacts are visible (dash-lined arrow). B High-resolution late-enhancement MRI image after injection of a fibrin-binding contrast agent. The thrombus can be noninvasively illustrated in the region of the distal LAD using a clear focal positive contrast. Subsequently the thrombus was confirmed using angiography. Adapted from [51].

formed blood vessels (angioneogenesis). Resulting from the subsequent phagocytosis by local macrophages, focal accumulation could occur in macrophages [55]. Successful noninvasive imaging of macrophages was first described by Schmitz et al. and Ruehm et al. using a rabbit model [56, 57]. Recently it was also demonstrated that iron oxide par- ticles can be used to assess macrophage load as well as monitor inflammation activity under therapy [58]. In recent years various techniques have been developed and evaluated to determine whether they could be used to display iron oxide-marked macrophages with positive contrast. Examples include the following methods: IRON (inversion-recovery with ON-resonant water suppression), GRASP (gradient acquisition for superparamagnetic particles) and SGM (susceptibility gradient mapping) ( $\bullet$ Fig.4) $[43,59,60]$. An alternative approach to detecting macrophages uses gadolinium-based micella which specifically bind with scavenger receptors expressed by activated macrophages and which exhibit high affinity for oxidized LDL. The signal intensity increase in atherosclerotic plaques measured after micella application using an $\mathrm{apoE}^{-/-}$mouse showed good correlation with the presence of macrophage-rich plaques ( $\bullet$ Fig. 5) [61]. Lipoprotein-based molecular probes are an additional means to specifically demonstrate macrophage-rich plaques. HDL particles modified for molecular imaging can overcome the endothelial barrier and have likewise been successfully tested in studies of atherosclerosis [62-64].

\section{Molecular probes and contrast agents for displaying an-} gioneogenesis

When atherosclerotic lesions develop, new vessels form in the vascular wall. This process, called neoangiogenesis, is primarily triggered by local hypoxic states [65]. Progredient neoangiogenesis has a direct connection to increased plaque instability. Activated endothelial cells of these newlyformed vessels express various surface proteins (e.g. $\alpha_{v \beta 3}$ ) which are not expressed by inactive endothelial cells in healthy tissue [37]. Two strategies to detect neoangiogenesis have been successfully developed and evaluated. One approach relies upon direct visualization of specific surface markers on endothelial cells of newly-formed vessels. Using gadolinium-based liposomes that bond specifically with $\alpha_{v \beta 3}$ integrin, local angioneogenesis has been successfully demonstrated on a rabbit model with atherosclerosis [37]. Alternatively, the increased blood flow resulting from neovascularization can be directly visualized and quantified through the utilization of dynamic contrast-enhanced MRI sequences [66]. In this case, clinically-approved contrast agents such as Gd-DTPA are suitable, as they effect a change in signal intensity in the target region across a wide dosage range as a function of their concentration. In order to quantify neoangiogenesis, changes in signal intensity are measured over time using dynamic T1-weighted sequences with high chronological resolution.

\section{Summary}

$\nabla$

In recent years cardiovascular MRI imaging has developed rapidly and the resulting technical advances enable reliable morphological representation and assessment of the vascular wall of coronary arteries as well as the carotid arteries. Various preclinical and clinical studies using molecular probes have shown that both macrophages and fibrin can be selectively visualized in experimental models and patients. Recently published experimental studies demonstrated that a newly developed elastin-specific contrast 

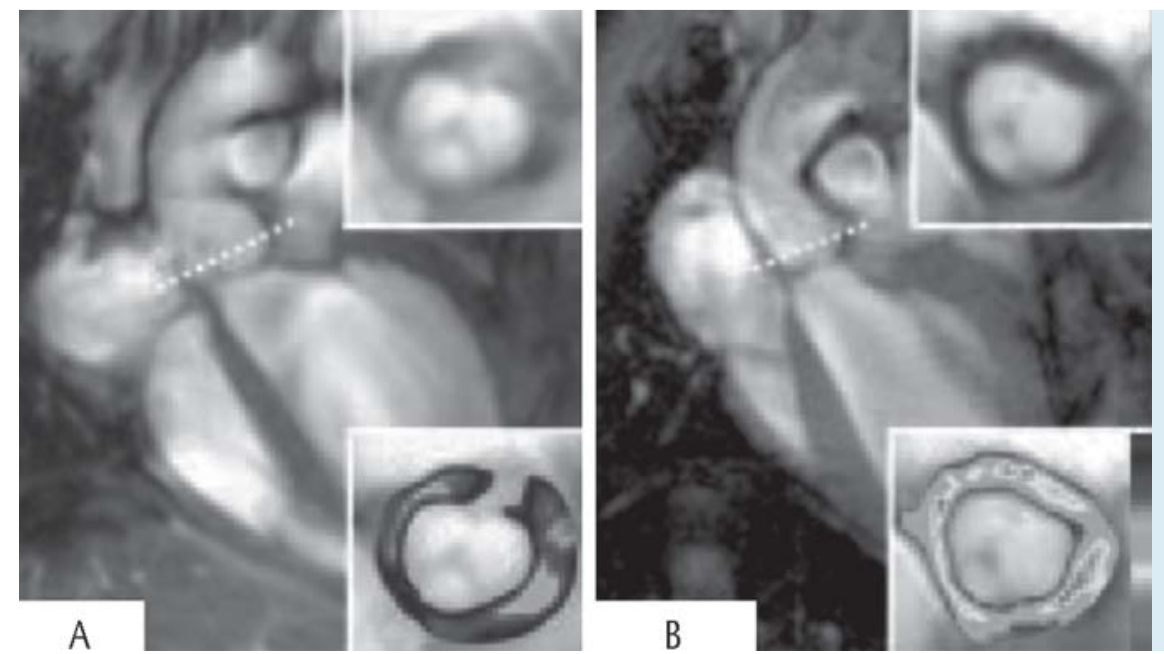

Fig. 3 In vivo detection and visualization of an early inflammatory alteration of the atherosclerotic vascular wall using a VCAM-1 specific MRI probe. The inflammatory process in early atherosclerotic vascular wall alteration was reproduced in the aortic root of apo $\mathrm{E}^{-1-}$ mice before $\mathbf{A}$ and after $\mathbf{B}$ the injection of VINP-28 probes, a specific marker for VCAM1. Significant reduction of signal intensity could be detected, visualized and quantified in transversal MRI images ( $T E=2.7$ and $4.7 \mathrm{~ms}$, TR $7.0-8.0 \mathrm{~ms}$ ). The significantly reduced contrast-noise ratio after application of the probe indicates the binding of the probe and the non-invasive VCAM- 1 expression by endothelial cells and macrophages. Adapted from [54].

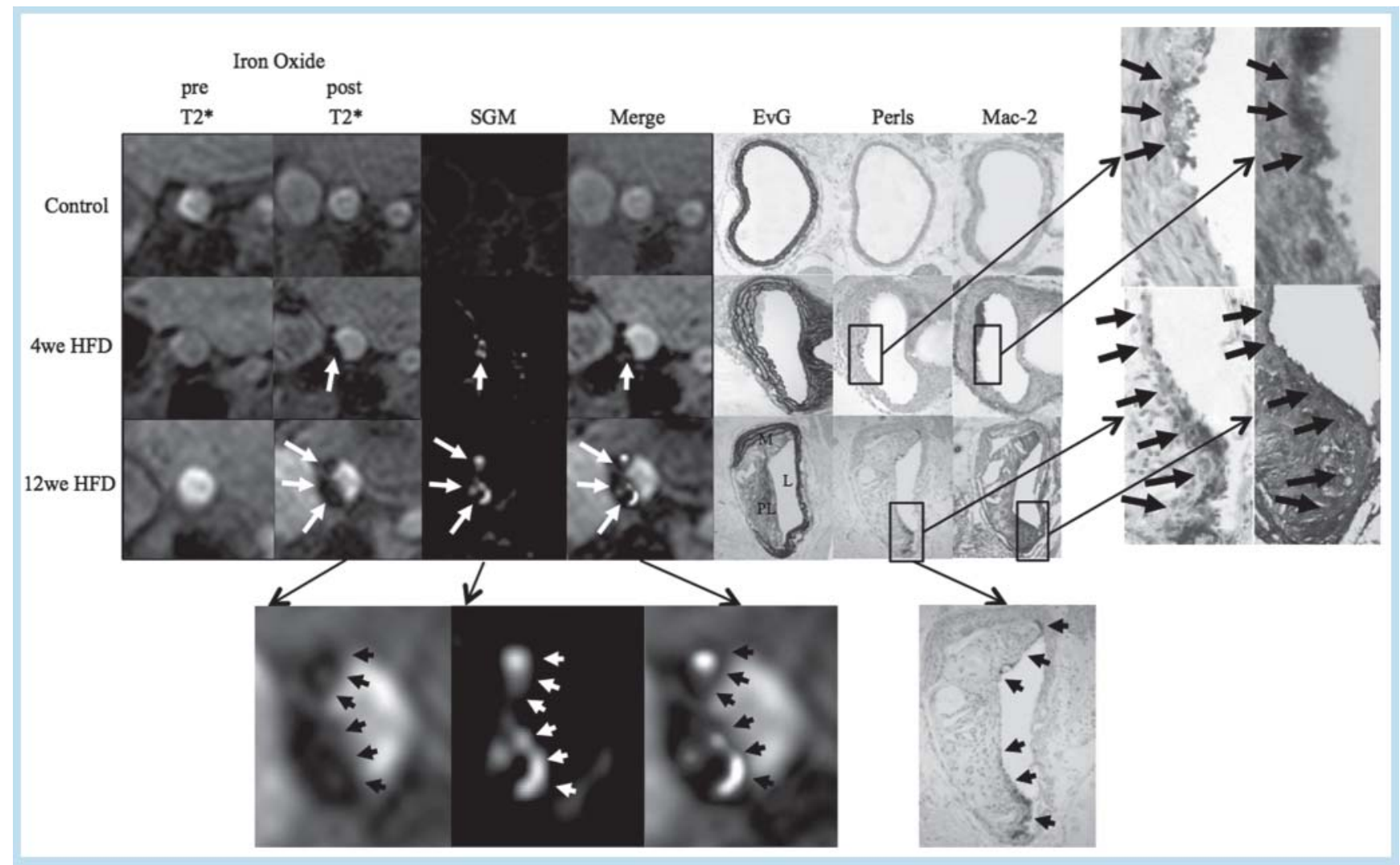

Fig. 4 Non-invasive evaluation of inflammation load in the course of atherosclerotic plaque development. This study showed that inflammation load can be visualized and quantified non-invasively using positive contrast. This study used a mouse model with atherosclerosis. In the control group no signal reduction or loss could be detected after injection of iron oxide. In the early stage of atherosclerotic plaque development, initial atherosclerotic vascular wall alterations could be detected after 4 weeks ( 2 nd row, 4 weeks HFD). In the periphery of the atherosclerotic plaques focal enrichment of iron oxide particles could be detected in vivo (Perls coloration). This enrichment could be sensitively and noninvasively quantified using SGM MRI. In advanced atherosclerotic plaques, significant enrichment of iron oxide particles could be detected as a signal reduction in $\mathrm{T} 2{ }^{*}$-weighted images in the SGM MRI (susceptibility gradient mapping MRI). The histological assessment confirmed the non-invasive measurements. The positive contrast generated by the SGM MRI enabled clear visualization of the enrichment of iron oxide particles in the plaque, and allowed quantification of the enrichment (mTm) in SG parameter maps. Adapted from [43]. 

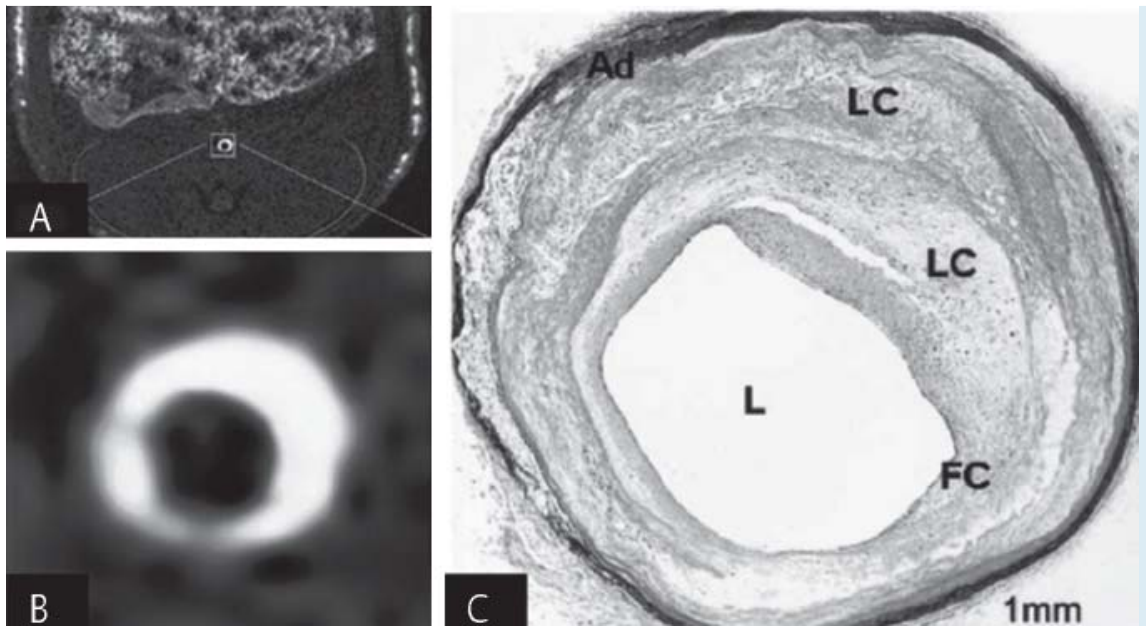

Fig. 5 Characterization of atherosclerotic plaques and application of gadofluorine. A, B T1-weighted MR image of the aorta in a rabbit model 24 hours after intravenous injection of gadofluorine. C: Corresponding histological image displaying the different components of atherosclerotic plaque. In this experimental study it was shown that gadofluorine can be successfully employed for the detection of lipid-rich atherosclerotic plaques. L: Lumen, Ad: Adventitia, LC: Lipid Core, FC: Fibrous Cap. Adapted from [46]. agent permits in vivo visualization and quantification of plaque load and plaque remodeling. Both factors represent independent predictors of future cardiac events. This molecular probe has been additionally tested successfully in a large animal subject with coronary remodeling. These promising results demonstrate the potential of molecular MRI probes for in vivo detection of early molecular and cellular alterations as well as for quantification of the therapeutic outcome. Biological characterization of atherosclerotic plaques could enable a distinction to be made between stable and unstable plaques, more accurate forecasting of cardiovascular events as well as improved therapy monitoring. In conclusion, within the context of atherosclerosis molecular MRI has contributed to the understanding of vascular biology and has demonstrated the potential to achieve more accurate clinical risk assessment. With further technical advances in MRI hardware and development of specific molecular probes, this technology could contribute to improving noninvasive diagnostics of atherosclerosis as well as therapy monitoring in clinical practice. In the future, rapid advances in cardiovascular MRI-based early diagnosis could significantly reduce total cardiovascular mortality.

\section{Affiliations}

Imaging Sciences, King's College London BHF Centre, Division of Imaging Science, Biomedical Research Centre of Guy's and St. Thomas' NHS Foundation Trust, London, UK, London

2 Heart Center Munich-Bogenhausen, Munich, Germany, Department of Cardiology and Intensive Care Medicine, Munich

Institute for Radiology, CCM, Charité, Berlin

${ }^{4}$ Department of Cardiology, CBF, Charité, Berlin

Institute for Radiology, CVK, Charité, Berlin

${ }^{6}$ Department of Nuclear Medicine, Charité, Berlin
}

\section{References}

1 Roger VL, Go AS, Lloyd-Jones DM et al. Heart disease and stroke statistics-2011 update: a report from the American Heart Association. Circulation 2011; 123: e18-e209

2 Ambrose JA, Tannenbaum MA, Alexopoulos D et al. Angiographic progression of coronary artery disease and the development of myocardial infarction. J Am Coll Cardiol 1988; 12: 56-62

3 Burke AP, Kolodgie FD, Farb A et al. Morphological predictors of arterial remodeling in coronary atherosclerosis. Circulation 2002; 105: 297 303

4 Varnava AM, Mills PG, Davies MJ. Relationship between coronary artery remodeling and plaque vulnerability. Circulation 2002; 105: 939 - 943

5 Glagov S, Weisenberg E, Zarins CK et al. Compensatory enlargement of human atherosclerotic coronary arteries. N Engl J Med 1987; 316: $1371-1375$

6 Rentrop KP. Thrombi in acute coronary syndromes: revisited and revised. Circulation 2000; 101: 1619-1626

7 Botnar RM, Stuber M, Kissinger KV et al. Noninvasive coronary vessel wall and plaque imaging with magnetic resonance imaging. Circulation 2000; 102: 2582-2587

8 Fayad ZA, Fuster V, Fallon JT et al. Noninvasive in vivo human coronary artery lumen and wall imaging using black-blood magnetic resonance imaging. Circulation 2000; 102: 506-510

9 Corti R, Fuster V, Fayad ZA et al. Effects of aggressive versus conventional lipid-lowering therapy by simvastatin on human atherosclerotic lesions: a prospective, randomized, double-blind trial with high-resolution magnetic resonance imaging. J Am Coll Cardiol 2005; 46: 106 112

10 Ibrahim T, Makowski MR, Jankauskas A et al. Serial contrast-enhanced cardiac magnetic resonance imaging demonstrates regression of hyperenhancement within the coronary artery wall in patients after acute myocardial infarction. JACC Cardiovasc Imaging 2009; 2: 580 588

11 Gore JC, Yankeelov TE, Peterson TE et al. Molecular imaging without radiopharmaceuticals? Journal of nuclear medicine: official publication, Society of Nuclear Medicine 2009; 50: 999-1007

12 Sato $Y$, Hatakeyama $K$, Marutsuka $K$ et al. Incidence of asymptomatic coronary thrombosis and plaque disruption: comparison of non-cardiac and cardiac deaths among autopsy cases. Thromb Res 2009; 124 : $19-23$

13 Libby P, Ridker PM, Hansson GrK. Progress and challenges in translating the biology of atherosclerosis. Nature $2011 ; 473$ : $317-325$

14 Krettek A, Sukhova GK, Libby P. Elastogenesis in human arterial disease: a role for macrophages in disordered elastin synthesis. Arterioscler Thromb Vasc Biol 2003; 23: 582 - 587

15 Kolodgie FD, Narula J, Yuan C et al. Elimination of neoangiogenesis for plaque stabilization: is there a role for local drug therapy? Journal of the American College of Cardiology 2007; 49: 2093 - 2101

16 Kolodgie FD, Gold HK, Burke AP et al. Intraplaque hemorrhage and progression of coronary atheroma. The New England journal of medicine 2003; 349: 2316-2325 
17 Narula J, Garg P, Achenbach S et al. Arithmetic of vulnerable plaques for noninvasive imaging. Nature Clinical Practice Cardiovascular Medicine 2008; 5: S2-S10

18 Stone GW, Maehara A, Lansky AJ et al. A prospective natural-history study of coronary atherosclerosis. The New England journal of medicine 2011; 364: 226-235

19 Burke AP, Farb A, Malcom GT et al. Effect of risk factors on the mechanism of acute thrombosis and sudden coronary death in women. Circulation 1998; 97: 2110-2116

20 Johnson RC, Leopold JA, Loscalzo J. Vascular calcification: pathobiological mechanisms and clinical implications. Circ Res 2006; 99: 10441059

21 Aikawa E, Nahrendorf M, Figueiredo JL et al. Osteogenesis associates with inflammation in early-stage atherosclerosis evaluated by molecular imaging in vivo. Circulation 2007; 116: 2841 - 2850

22 Proudfoot D, Skepper JN, Hegyi L et al. Apoptosis regulates human vascular calcification in vitro: evidence for initiation of vascular calcification by apoptotic bodies. Circ Res 2000; 87: 1055-1062

23 Tyson KL, Reynolds JL, McNair R et al. Osteo/chondrocytic transcription factors and their target genes exhibit distinct patterns of expression in human arterial calcification. Arteriosclerosis, thrombosis, and vascular biology 2003; 23: 489-494

24 Virmani R, Ladich ER, Burke AP et al. Histopathology of carotid atherosclerotic disease. Neurosurgery 2006; 59: S219-S227

25 Johnson GA, Benveniste H, Black RD et al. Histology by magnetic resonance microscopy. Magn Reson Q 1993; 9: 1- 30

26 Schmitz SA, Coupland SE, Gust R et al. Superparamagnetic iron oxideenhanced MRI of atherosclerotic plaques in Watanabe hereditable hyperlipidemic rabbits. Invest Radiol 2000; 35: 460-471

27 Caravan P, Ellison JJ, McMurry TJ et al. Gadolinium(III) Chelates as MRI Contrast Agents: Structure, Dynamics, and Applications. Chem Rev 1999; 99: 2293-2352

28 Weinmann HJ, Brasch RC, Press WR et al. Characteristics of gadoliniumDTPA complex: a potential NMR contrast agent. Am J Roentgenol Am J Roentgenol 1984; 142: 619-624

29 Nazarpoor $M$. Effects of inversion and saturation times on relationships between contrast agent concentrations and signal intensities of T1-weighted magnetic resonance images. Radiol Phys Technol 2010; 3: $120-126$

30 Mugler JP 3rd, Brookeman JR. Theoretical analysis of gadopentetate dimeglumine enhancement in T1-weighted imaging of the brain: comparison of two-dimensional spin-echo and three-dimensional gradient-echo sequences. J Magn Reson Imaging 1993; 3: 761 - 769

31 Kok M, Hak S, Mulder W et al. Cellular compartmentalization of internalized paramagnetic liposomes strongly influences both $\mathrm{T}$ 1and $\mathrm{T}$ 2relaxivity. Magnetic Resonance in Medicine 2009; 61: 1022 - 1032

32 Caravan P, Farrar CT, Frullano $L$ et al. Influence of molecular parameters and increasing magnetic field strength on relaxivity of gadolinium- and manganese-based T1 contrast agents. Contrast Media Mol Imaging 2009; 4: 89-100

33 Nivorozhkin AL, Kolodziej AF, Caravan P et al. Enzyme-Activated Gd(3+) Magnetic Resonance Imaging Contrast Agents with a Prominent Receptor-Induced Magnetization Enhancement. Angew Chem Int Ed Engl 2001; 40: 2903-2906

34 Caravan $P$. Strategies for increasing the sensitivity of gadolinium based MRI contrast agents. Chemical Society Reviews 2006; 35: 512

35 Botnar RM, Buecker A, Wiethoff AJ et al. In vivo magnetic resonance imaging of coronary thrombosis using a fibrin-binding molecular magnetic resonance contrast agent. Circulation 2004; 110: $1463-$ 1466

36 Flacke S, Fischer S, Scott MJ et al. Novel MRI contrast agent for molecular imaging of fibrin: implications for detecting vulnerable plaques. Circulation 2001; 104: 1280-1285

37 Winter PM, Morawski AM, Caruthers SD et al. Molecular imaging of angiogenesis in early-stage atherosclerosis with alpha(v)beta3-integrintargeted nanoparticles. Circulation 2003; 108: 2270-2274

38 Weissleder R, Elizondo G, Wittenberg J et al. Ultrasmall superparamagnetic iron oxide: characterization of a new class of contrast agents for MR imaging. Radiology 1990; 175: 489-493

39 Farrar CT, Dai G, Novikov $M$ et al. Impact of field strength and iron oxide nanoparticle concentration on the linearity and diagnostic accuracy of off-resonance imaging. NMR Biomed 2008; 21: 453-463

40 Ferrucci JT, Stark DD. Iron oxide-enhanced MR imaging of the liver and spleen: review of the first 5 years. Am J Roentgenol Am J Roentgenol 1990; 155: $943-950$
41 Small WC, Nelson RC, Bernardino ME. Dual contrast enhancement of both T1- and T2-weighted sequences using ultrasmall superparamagnetic iron oxide. Magn Reson Imaging 1993; 11: 645-654

42 Makowski MR, Wiethoff AJ, Blume U et al. Assessment of atherosclerotic plaque burden with an elastin-specific magnetic resonance contrast agent. Nat Med 2011; 17: 383-388

43 Makowski MR, Varma G, Wiethoff A et al. Non-Invasive Assessment of Atherosclerotic Plaque Progression in ApoE-/- Mice Using Susceptibility Gradient Mapping. Circ Cardiovasc Imaging 2011; DOI: 10.1161/ CIRCIMAGING.110.957209

44 von Bary C, Makowski M, Preissel A et al. MRI of Coronary Wall Remodeling in a Swine Model of Coronary Injury Using an Elastin-Binding Contrast Agent. Circ Cardiovasc Imaging 2011; 4: 147-155

45 Meding J, Urich M, Licha K et al. Magnetic resonance imaging of atherosclerosis by targeting extracellular matrix deposition with gadofluorine M. Contrast Media Mol Imaging 2007; 2: 120-129

46 Sirol M, Itskovich VV, Mani $V$ et al. Lipid-rich atherosclerotic plaques detected by gadofluorine-enhanced in vivo magnetic resonance imaging. Circulation 2004; 109: 2890-2896

47 Sirol M, Moreno P, Purushothaman K et al. Increased Neovascularization in Advanced Lipid-Rich Atherosclerotic Lesions Detected by Gadofluorine-M-Enhanced MRI: Implications for Plaque Vulnerability. Circulation: Cardiovascular Imaging 2009; 2: 391 - 396

48 Ronald JA, Chen Y, Belisle AJ et al. Comparison of gadofluorine-M and Gd-DTPA for noninvasive staging of atherosclerotic plaque stability using MRI. Circ Cardiovasc Imaging 2009; 2: 226-234

49 Tavora F, Cresswell N, Li L et al. Immunolocalisation of fibrin in coronary atherosclerosis: implications for necrotic core development. Pathology 2010; 42: 15-22

50 Botnar RM, Perez AS, Witte $S$ et al. In vivo molecular imaging of acute and subacute thrombosis using a fibrin-binding magnetic resonance imaging contrast agent. Circulation 2004; 109: $2023-2029$

51 Spuentrup E, Buecker A, Katoh M et al. Molecular Magnetic Resonance Imaging of Coronary Thrombosis and Pulmonary Emboli With a Novel Fibrin-Targeted Contrast Agent. Circulation 2005; 112: 1594-1600

52 Spuentrup E, Botnar RM, Wiethoff A et al. MR imaging of thrombi using EP-2104R, a fibrin specific contrast agent: initial results in patients. European Radiology 2008; 18: 1995-2005

53 Cybulsky MI, Gimbrone MA Jr. Endothelial expression of a mononuclear leukocyte adhesion molecule during atherogenesis. Science 1991; 251: $788-791$

54 Nahrendorf M, Jaffer F, Kelly K et al. Noninvasive Vascular Cell Adhesion Molecule-1 Imaging Identifies Inflammatory Activation of Cells in Atherosclerosis. Circulation 2006; 114: 1504-1511

55 Kooi ME, Cappendijk VC, Cleutjens KB et al. Accumulation of ultrasmall superparamagnetic particles of iron oxide in human atherosclerotic plaques can be detected by in vivo magnetic resonance imaging. Circulation 2003; 107: 2453-2458

56 Ruehm SG, Corot C, Vogt P et al. Magnetic resonance imaging of atherosclerotic plaque with ultrasmall superparamagnetic particles of iron oxide in hyperlipidemic rabbits. Circulation 2001; 103: 415-422

57 Schmitz SA, Coupland SE, Gust R et al. Superparamagnetic iron oxideenhanced MRI of atherosclerotic plaques in Watanabe hereditable hyperlipidemic rabbits. Investigative radiology 2000; 35: 460-471

58 Morishige $K$, Kacher DF, Libby $P$ et al. High-resolution magnetic resonance imaging enhanced with superparamagnetic nanoparticles measures macrophage burden in atherosclerosis. Circulation 2010; 122: $1707-1715$

59 Korosoglou G, Weiss RG, Kedziorek DA et al. Noninvasive detection of macrophage-rich atherosclerotic plaque in hyperlipidemic rabbits using "positive contrast" magnetic resonance imaging. Journal of the American College of Cardiology 2008; 52: 483-491

60 Mani V, Briley-Saebo KC, Itskovich VV et al. Gradient echo acquisition for superparamagnetic particles with positive contrast (GRASP): sequence characterization in membrane and glass superparamagnetic iron oxide phantoms at $1.5 \mathrm{~T}$ and 3T. Magn Reson Med 2006; 55: $126-135$

61 Amirbekian V, Lipinski MJ, Briley-Saebo KC et al. Detecting and assessing macrophages in vivo to evaluate atherosclerosis noninvasively using molecular MRI. Proc Natl Acad Sci U S A 2007; 104: 961 - 966

62 Frias JC, Ma Y, Williams KJ et al. Properties of a versatile nanoparticle platform contrast agent to image and characterize atherosclerotic plaques by magnetic resonance imaging. Nano Lett 2006; 6: 2220-2224 
63 Cormode DP, Briley-Saebo KC, Mulder WJ et al. An ApoA-I mimetic peptide high-density-lipoprotein-based MRI contrast agent for atherosclerotic plaque composition detection. Small 2008; 4: 1437-1444

64 Chen W, Vucic E, Leupold E et al. Incorporation of an apoE-derived lipopeptide in high-density lipoprotein MRI contrast agents for enhanced imaging of macrophages in atherosclerosis. Contrast Media Mol Imaging 2008 ; $3: 233-242$
65 Purushothaman KR, Sanz J, Zias E et al. Atherosclerosis neovascularization and imaging. Curr Mol Med 2006; 6: 549-556

66 Kerwin WS, O'Brien KD, Ferguson MS et al. Inflammation in carotid atherosclerotic plaque: a dynamic contrast-enhanced MR imaging study. Radiology 2006; 241: 459-468 